\title{
Effects of a Restrictive Antibiotic Policy on Antibiotic Usage and Staphylococcus Aureus Resistance
}

\author{
Qiu Fan * \\ Department of pharmacy \\ Yancheng Medicine College \\ Yancheng, China \\ e-mail: echo_qf@163.com \\ * Corresponding Author \\ Jin Hao \\ Clinical Laboratory \\ Yancheng 3th people hospital \\ Yancheng, China \\ e-mail: Haojin@126.com
}

\author{
Meng Li \\ Department of science and technology \\ Yancheng Medicine College \\ Yancheng, China \\ e-mail: li97101@ycmc.edu.cn \\ Chen Jun \\ Department of student management \\ Yancheng Medicine College \\ Yancheng, China \\ e-mail: 199293@ycmc.edu.cn
}

Keywords: Antibiotic; Rational use; antibiotic policy; antibiotic resistance; Staphylococcus aureus

\begin{abstract}
Objective: This paper aims to report the survey of antimicrobial usage and resistance to Staphylococcus aureus in a local hospital under the restrictive antibiotic policy in China.

Methods: We conducted the analysis using retrospective data from 2011 to 2014. The hospital provided data concerning hospital characteristics and infection control policies. The data concerning antibiotic utilization were collected from hospital pharmacy records and reports. The antimicrobial susceptibility test were monitored and recorded in hospital microbiology laboratory.

Results: Due to the restrictive antibiotic policy implemented in 2011, antibiotic prescription, antibiotic consumption, antibiotic procurement and prophylactic use of antibiotics in clean operations were all declined. The submission rate of microbial test in antimicrobial therapy increased, but the blood and other sterile site specimen need to be further applied. The broad spectrum antimicrobials and last line drugs still keep a regular susceptibility to Staphylococcus aureus. The factors of high age, long stay in hospital, long time for antibiotic usage and invasive operation were all related to antibiotic resistance.

Conclusion: The intervention of antibiotic policy was effective to ensure the rational use of antibiotics.
\end{abstract}

\section{Introduction}

Antimicrobial resistance is a serious public health challenge. Today's societies face the global threat of antibiotic resistant "superbugs," organisms such as methicillin-resistant Staphylococcus aureus (MRSA), vancomycin-insensitive Staphylococcus aureus (VISA) and vancomycin-resistant Staphylococcus aureus (VRSA) [1]. Carriers of Staphylococcus aureus are at a high risk of developing invasive infections [2]. The clinical spectrum of infectious syndromes associated with Staphylococcus aureus ranges from a commensal state to serious overwhelming infections like skin and soft tissue infections, endovascular infections, necrotizing pneumonia, septic arthritis, endocarditis, osteomyelitis and sepsis [3,4].

As a response to these serious infections, standardized management of antibiotics use were necessary to decrease irrational use of antimicrobial agents. In 2011, the Chinese Ministry of Health adjusted the reliance on the professional strategies and launched a special campaign to reorganize the 
rational use of antimicrobials in healthcare settings [5]. Hospitals were required to set up a drug therapeutic committee to facilitate the rational use of drugs, develop audit and inspection systems, investigate and reassign responsibility to hospital management staff [6].

Prospective surveillance programs were also set up in Yancheng 3th people hospital in 2011. This survey mainly focuses on the surveillance of Staphylococcus aureus, monitor the impact of policy on resistance rate and find out the factors with the influence.

\section{Method}

This study was performed at the third People Hospital, an 800-bed, tertiary-care teaching hospital affiliated with Southeast University in Yancheng, China. The strict antibiotic policy was implemented in this hospital in 2011. The usage and cost of antibiotics for inpatients was surveyed through case histories and hospital information system (HIS). In clinical laboratory, antimicrobial Susceptibility Testing was performed using the disk diffusion method and susceptibility profiles were determined using zone diameter interpretive criteria, as recommended by the Clinical and Laboratory Standards Institute (CLSI) in 2011 (M100-S21). The data were recorded from 2011 to 2014.

\section{Results}

The Main Results of Important Index Investigated. Follow the instructions of the campaign protocol launched by the Chinese Ministry of Health, we have reached the target. The main index and the results of survey were listed in Table 1 . The target for antibiotic prescription was set at less than $60 \%$ for hospitalized patients. The data have declined to $41.5 \%$ in 2014 in our hospital. The antibiotic usage was standardized based on the WHO defined daily doses (DDDs) per 100 bed days and should be less than 40 DDDs per 100 patient days. Prophylactic use rate of antibiotics in clean operations drop sharply from $76.98 \%$ in 2011 to $15.66 \%$ in 2014 . The rate of microbial test of antimicrobial therapy increased year by year and reached to $62.22 \%$ in 2014 . Antibiotic procurement restrict is another measure to prevent the antibiotic abuse, the number of agent should be less than 50 or 35 in secondary and tertiary hospitals, respectively. Our hospital procured 42 antibiotic agents in 2014 as a secondary hospital.

Table 1 Usage of antibiotics in hospitalized patients from 2011 to 2014

\begin{tabular}{llllll}
\hline \multicolumn{1}{c}{ Index } & Standard & 2011 & 2012 & 2013 & 2014 \\
\hline Antibiotic prescription for hospitalized patients (\%) & $\leq 60$ & 67.91 & 53.91 & 42.01 & 41.50 \\
$\begin{array}{l}\text { Defined daily dose per 100 patient days } \\
\text { Prophylactic use of antibiotics in clean operations (\%) }\end{array}$ & $\leq 40$ & 60.93 & 43.22 & 40.08 & 37.52 \\
$\begin{array}{l}\text { The submission rate of microbial test of antimicrobial } \\
\text { therapy (\%) }\end{array}$ & $\geq 30$ & 76.98 & 19.91 & 18.90 & 15.66 \\
$\begin{array}{l}\text { The number of antibiotic procurement in secondary } \\
\text { hospitals }\end{array}$ & $\leq 50$ & 82 & 53.51 & 55.90 & 62.22 \\
\end{tabular}

The Analysis of Different Culture Specimen from Clinidal Test Samples. During the four year, 815 isolates were collected and identified as Staphylococcus aureus strains which represented 6.5\% of all the isolated strains. The strains were cultured from sputum, secretions and pus, blood, urine and others (Fig.1). Skin and soft tissue infections caused by Staphylococcus aureus are commonly encountered [7], so the sputum and secretion/pus specimen accounts a large proportion. The antibiotic protocols suggested improve the proportion of the blood and other sterile site specimen as the basis for the choose antibiotics [8]. Because detecting the microorganism in blood is more accurate and helpful for the Doctor's diagnose than in sputum, though the sputum culture specimen is very easy to obtain. The proportion of different specimen were showed in Fig. 1, there still are many space to increase the proportion of blood and other sterile site specimen. 


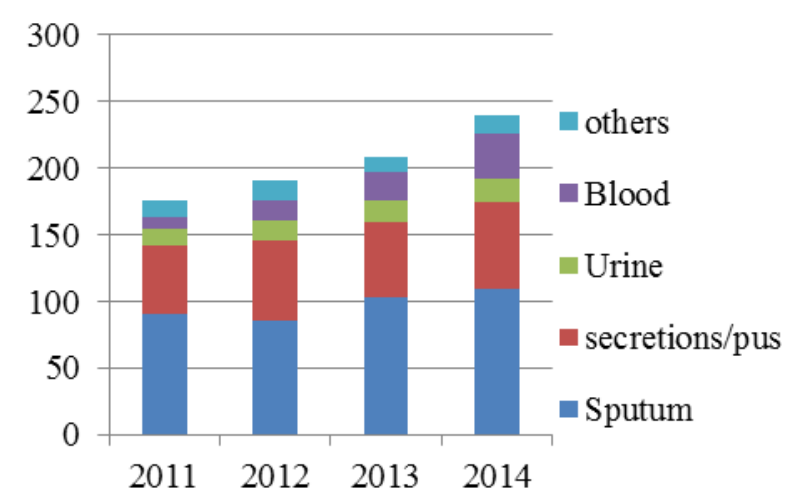

Figure 1. the number of different culture specimen from 2011 to 2014

The Results of Susceptibility Rates (\%) of Staphylococcus Aureus Against Antimicrobial Agents. Table 2 listed the results of susceptibility tests of Staphylococcus aureus strains against antimicrobial agents. Quinupristin/Dafoeleptin and Tigecycline were introduced in our hospital in 2012, there wasn't data in 2011. As the new powerful antibiotics, they are used only in intensive care unit (ICU). Linezolid and vancomycin keep very sensitive to S. aureus in our hospital as the last line therapeutic agents for many years to cure MRSA infections, though there were reports about the resistance to Linezolid and vancomycin in the world [8]. For the strict supervision, the susceptibility rate of nitrofurantoin and rifampin are over $90 \%$, the susceptibility rate of moxifloxacin rised to $84.62 \%$. The laboratory marker of MRSA is oxacillin resistance [9]. The susceptibility rate (48.08\%) to oxacillin indicates $51.92 \%$ Staphylococcus aureus were resistant to oxacillin, that is MRSA. Though the broad spectrum antimicrobials were mostly used, the susceptibility rates were mostly above 50\% such as Gentamicin, tetracycline and levofloxacin. Most antibiotic agents susceptibility to Staphylococcus aureus rised during the three years, but the susceptibility rate of clindamycin and erythromycin decreased, that of ciprofloxacin and penicillin g fluctuated, this may be due to the antibiotic consumption compensation effect when the usage of some antibiotic agent decreased, some others increased.

Analysis of Risk Factors for Staphylococcus Aureus Infection. A random sample group of 160 Staphylococcus aureus strains were isolated from 815 cases. They are sorted by age, gender, duration of antibiotics usage, duration of hospital stay and invasive operation. Invasive operations mainly contain tracheotomy, indwelling gastric tube, mechanical ventilation and drainage tube. Multiply antibiotic resistance rate was the ratio of multiple drug resistance cases and case number.

The results (Table 3) showed high age, long stay in hospital, long time for antibiotic usage and invasive operation were all related to antibiotic resistance. People staying in hospital over a month were highly likely infected by multidrug-resistant bacteria according to multiply antibiotic resistance rate. Exactly the results reminds us it should pay more attention to people who are over 60 years, male, using antibiotics over two weeks, in invasive surgery. 
Table 2 Usage of antibiotics in hospitalized patients

\begin{tabular}{ccccc}
\hline Antibiotic & 2011 & 2012 & 2013 & 2014 \\
\hline Quinupristin / Dafoeleptin & - & 100.00 & 99.17 & 100.00 \\
Tigecycline & - & 100.00 & 100.00 & 100.00 \\
Linezolid & 99.05 & 99.12 & 100.00 & 100.00 \\
Vancomycin & 96.19 & 96.55 & 97.50 & 98.08 \\
Nitrofurantoin & 96.02 & 95.65 & 99.17 & 96.15 \\
Rifampin & 91.40 & 96.55 & 97.48 & 92.31 \\
Moxifloxacin & 56.99 & 75.00 & 97.50 & 84.62 \\
Oxacillin & 77.14 & 47.41 & 59.17 & 48.08 \\
Gentamicin & 58.33 & 68.10 & 94.17 & 86.54 \\
Ampicillin/Sulbactam & 73.30 & 86.10 & 100.00 & 98.00 \\
Tetracycline & 50.00 & 83.62 & 94.17 & 86.54 \\
Compound Sulfamethoxazole & 55.56 & 71.55 & 91.67 & 84.62 \\
Levofloxacin & 41.67 & 57.76 & 82.50 & 57.69 \\
Clindamycin & 78.38 & 35.34 & 45.83 & 21.15 \\
Ciprofloxacin & 51.02 & 54.31 & 80.00 & 55.77 \\
Erythromycin & 21.90 & 24.14 & 45.00 & 15.38 \\
Penicillin G & 13.33 & 1.72 & 27.50 & 5.77 \\
\hline & & & &
\end{tabular}

Table 3 Analysis of risk factors for Staphylococcus aureus infection

\begin{tabular}{ccccc}
\hline \multicolumn{2}{c}{ Correlative factor } & $\begin{array}{c}\text { Case number } \\
\text { (ratio) }\end{array}$ & $\begin{array}{c}\text { Multiply antibiotic } \\
\text { resistance rate }(\%)\end{array}$ & P-value \\
\hline age & $<20$ & $8(5.1 \%)$ & 11.6 & \\
& $20 \sim 39$ & $26(16.5 \%)$ & 19.8 & 0.01 \\
gender & $40 \sim 59$ & $46(28.7 \%)$ & 45.7 & \\
& $>60$ & $82(51.2 \%)$ & 49.4 & 0.85 \\
Duration of & male & $102(63.7 \%)$ & 39.2 & \\
antibiotics usage & female & $58(36.4 \%)$ & 41.3 & 0.01 \\
Duration of & $<14 \mathrm{~d}$ & $58(36.2 \%)$ & 10.3 & \\
hospital stay & $14 \mathrm{~d} 28 \mathrm{~d}$ & $64(40.0 \%)$ & 46.9 & 0.01 \\
& $>28 \mathrm{~d}$ & $38(23.7 \%)$ & 74.7 & \\
Invasive & $<14 \mathrm{~d}$ & $58(36.7 \%)$ & 16.9 & 31.1 \\
operation & $15 \sim 30 \mathrm{~d}$ & $45(28.1 \%)$ & 70.4 & 0.00 \\
& $>30 \mathrm{~d}$ & $56(35.0 \%)$ & 64.0 & \\
\hline
\end{tabular}

\section{Discussion and conclusion}

It has been generally recognized that the prevalence of bacterial resistance among bacteria is an unavoidable consequence of irrational antibiotic use and is positively linked to the use of antibacterial drugs [10]. Controlling the spread of these organisms is a major public health challenge and often involves labor-intensive, protracted efforts. In our hospital, we have an antibiotic administrative group chaired by the president, set targets for antimicrobial management, investigated responsibility to hospital management staff who violate rational use policies. The results of this survey showed all index investigated in 2014 were better than that in 2011, it indicate that a rational and strict antibiotic 
policy is of great effectiveness for the rational use of these agents. Continuous monitoring is needed to understand trends of antibiotics resistance and to act on these where necessary.

\section{Acknowledgment}

Supported by a project grant from Yancheng medical science and technology development project (Grand No.YK2013056)

\section{References}

[1] M. A. Lawson, "The Antibiotic Resistance Problem Revisited," American Biology Teacher (National Association of Biology Teacher), vol.70, 2008, pp. 405-410J. Clerk Maxwell, A Treatise on Electricity and Magnetism, 3rd ed., vol. 2. Oxford: Clarendon, 1892, pp.68-73

[2] V. P. Vera, M. Miro, P. Goran and B.R. Ljiljana, "Antimicrobial utilization and bacterial resistance at three different hospitals," European Journal of Epidemiology, vol. 17, Aug. 2001, pp. 375-383

[3] P.G.D. lennie, S.C.Cooper, G. Herman, M.K. Surbhi, J.L.W. Rob, G. Marek, et al "Iinterventions to reduce colonization and transmission of antimicrobial - resistance bacteria in intensive care units: an interrupted time series study and cluster randomized trial, Lancet infect dis, vol. 14, 2014, pp. 31-39

[4] P. Vaidya, G. Pawar, N. Krishnamurthy, "Community acquired MRSA infections-Three recent cases and an overview of CA MRSA infections," Pediatric Infectious Disease, vol. 7, 2015, pp. 8-12

[5] X. Yonghong, Z. Jing, Z. Beiwen, Z. Lina, L. Sujuan, L. Lanjuan, "Changes in Chinese Policies to Promote the Rational Use of Antibiotics," Plos Medicine, vol.10, Nov. 2013, pp.331-343

[6] H. M. Kwint, P.D. van der Linden, M.M.B. Roukens and S. Natsch, "Intensification of antibiotic use within acute care hospitals in the Netherlands," J Antimicrob Chemother, vol.67, May. 2012, pp.2283-2288

[7] China MOH (2011) Protocol for special campaign of antibiotic administrative in healthcare institutions. Ministry of Health, May 8, 2011 Beijing

[8] Y. Muraki, M. Kitamura, Y. Maeda, T. Kitahara, T. Mori, H. Ikeue, et al "Nationwide surveillance of antimicrobial consumption and resistance to Pseudomonas aeruginosa isolates at 203 Japanese hospitals in 2010" Infection, vol.41, Mar. 2013, pp.415-423

[9] Y.X. Liew, P.Krishnan, C.L. Yeo, T.Y. Tan, S.Y. Lee, W.P. Lim, W.Lee, L.Y. Hsu, "Surveillance of broad-spectrum antibiotic prescription in Singaporean Hospitals: A 5-year Longitudinal Study, " Plos one, vol.6, Dec. 2011, pp.Doi:10.1371/journal.pone.0028751.

[10] L. Reynolds, M. Mckee, "Factors influencing antibiotic prescribing in China: an exploratory analysis," Health Policy, vol.90, 2009, pp.32-36. 